\title{
Relativistic description of valence energy losses in the interaction of fast electrons with clusters of dielectrics: Multiple-scattering approach
}

\author{
F. J. García de Abajo* \\ Materials Sciences Division, Lawrence Berkeley National Laboratory, Berkeley, California 94720
}

(Received 26 January 1999)

\begin{abstract}
A fully relativistic description of valence energy losses suffered by fast electrons passing near finite clusters of arbitrarily disposed dielectric objects is presented using an accurate technique suited to solve Maxwell's equations. The method is based upon an expansion of the electromagnetic field in terms of multipoles around each of the objects of the cluster. Multiple elastic scattering of those multipole expansions is then performed until convergence is achieved. The energy loss, obtained from the induced electric field acting back on the electron, is computed in a time proportional to the square of the number of objects in the cluster, $N^{2}$. Numerical examples are presented for various clusters formed by $N=1-198$ homogeneous spheres made of $\mathrm{SiO}_{2}$ and $\mathrm{Al}$, and also for clusters of $\mathrm{Si}$ spheres coated with $\mathrm{SiO}_{2}$. Both relativistic effects and the interaction between the constituents of the cluster are shown to be of primary importance in the understanding of the position and magnitude of the features exhibited by the calculated electron-energy-loss spectra. [S0163-1829(99)00631-1]
\end{abstract}

\section{INTRODUCTION}

In a scanning transmission electron microscope (STEM), it is possible to focus a $100-300-\mathrm{keV}$ electron beam on a spot of a diameter below $0.5 \mathrm{~nm}$ at the position of the specimen and to study the energy-loss spectra of transmitted electrons to yield information about local chemical and electronic structure. The high-energy part of the loss spectra (i.e., energy losses above $50 \mathrm{eV}$ ) is associated to the excitation of deep levels, which are spatially localized in general and permit one to observe details directly with lateral resolution on the sub- $\AA$ scale in the finest versions of the STEM. ${ }^{1}$ The low-energy part relies on the more probable valence losses, allowing one to image the sample while causing smaller damage. ${ }^{2-7}$ Valence excitations are connected to delocalized modes of plasmonic nature that involve induced currents and charges distributed along relatively large regions of the sample interfaces. Unfortunately, their interpretation is not direct, and requires comparing theory and experiment.

The number of geometries that have been successfully subjected to analytical examination is small. Those are in general simple surfaces like planes, ${ }^{8-11}$ isolated cylinders, ${ }^{12}$ coupled cylinders, ${ }^{13}$ parabolic wedges,${ }^{14}$ isolated spheres, ${ }^{15-19}$ two coupled spheres, ${ }^{20}$ spheroids, ${ }^{21}$ and the combination of a sphere or a hemisphere with a plane..$^{22-24} \mathrm{In}$ addition, effective-medium theories have been applied in the case of random systems. ${ }^{25-28}$

More recently, a numerical solution of Poisson's equation based upon the boundary element method has permitted us to simulate nonrelativistic electron-energy-loss spectra (EELS) for more complex geometries by self-consistently solving the induced charge density distributed on the interfaces of the sample. ${ }^{29-33}$ This approach has been applied to translationally invariant interfaces ${ }^{31-33}$ and axially symmetric interfaces $^{29-31}$ of otherwise arbitrary shape.

Relativistic effects, sometimes beyond mere corrections, are relevant in this context due to both the large electron velocities employed in the STEM $(\mathrm{V}>0.5 c)$ and the effects of retardation in the electromagnetic signal involved in interface modes extended over large interface areas. Nevertheless, only few of the analytical studies noted above relied on relativistic descriptions. This is the case of planar surfaces $^{10,11}$ and isolated cylinders ${ }^{12}$ and spheres. ${ }^{19}$

Numerical methods have also been employed in relativistic calculations of EELS for composite structures. In this sense, the transfer-matrix approach developed by Pendry and co-workers has permitted us to obtain energy-loss spectra in periodic systems. ${ }^{34-36}$ Recently, the boundary element method has been extended to solve Maxwell's equations for fast electrons moving near arbitrarily shaped interfaces, making use of boundary charges and currents to reduce the threedimensional Maxwell equations to two-dimensional surface integral equations. ${ }^{37}$ This approach has proved to be useful in yielding fully relativistic calculations of EELS for either axially symmetric interfaces ${ }^{37,38}$ or translationally invariant interfaces. ${ }^{38}$ Still, additional techniques are needed in order to deal with large clusters of disordered dielectric objects.

The purpose of this work is to reduce the problem of calculating the energy loss of fast electrons in the presence of a cluster of dielectric objects to the individual scattering properties of each of the constituents of the cluster. The numerical examples offered below are limited to homogeneous spheres and coated spheres, for which the scattering matrices are given by analytical expressions. ${ }^{39,19}$ The present formalism can be equally applied to more complex objects regardless of their composition, provided one knows their scattering matrices.

The theory of multiple scattering of radiation is briefly summarized in Sec. II. The application to the simulation of EELS is given in Sec. III. Section IV is devoted to discussing some numerical examples for clusters of different sizes. Finally, the main conclusions are presented in Sec. V. Gaussian atomic units (a.u., that is, $e=m=\hbar=1$ ) will be used from now on, unless otherwise specified. 


\section{MULTIPLE-SCATTERING APPROACH TO THE ELECTROMAGNETIC PROBLEM}

The solution of the electromagnetic field in the presence of a cluster of dielectric objects is expressed here in terms of the multiple elastic scattering of multipole expansions (MESME) around the objects of the cluster. A more detailed description of this formalism is given elsewhere. ${ }^{40}$

In a region of space free of external charges and currents, the electric field has a pure transverse nature, and thus it can be written in frequency space $\omega$ as ${ }^{39}$

$$
\mathbf{E}=\mathbf{L}_{\alpha} \psi_{\alpha}^{M}-\frac{i}{k} \nabla \times \mathbf{L}_{\alpha} \psi_{\alpha}^{E},
$$

where $k=\omega / c, \mathbf{L}_{\alpha}=-i\left(\mathbf{r}-\mathbf{r}_{\alpha}\right) \times \nabla$ is the orbital angular momentum operator relative to the position $\mathbf{r}_{\alpha}$, and $\psi_{\alpha}^{M}$ and $\psi_{\alpha}^{E}$ are magnetic and electric scalar functions. If the region under consideration is homogeneous and described by its local dielectric function $\epsilon_{j}$ and magnetic permeability $\mu_{j}$, one finds that, upon insertion of Eq. (1) into Maxwell's equations, the scalar functions satisfy the wave equation

$$
\left(\nabla^{2}+k_{j}^{2}\right) \psi=0,
$$

where $k_{j}=k \sqrt{\epsilon_{j} \mu_{j}}$ (the square root is chosen here to have a non-negative imaginary part). Consequently, every external field has to be made up of spherical plane waves with no net energy flux through any closed surface, and, therefore,

$$
\psi_{\alpha}^{\mathrm{ext}}(\mathbf{r})=\sum_{L} j_{L}\left[k_{j}\left(\mathbf{r}-\mathbf{r}_{\alpha}\right)\right] \psi_{\alpha, L}^{\mathrm{ext}}
$$

where $L=(l, m), j_{L}(\mathbf{u})=i^{l} j_{l}(|\mathbf{u}|) Y_{L}(\hat{\mathbf{u}})$ is one of the noted spherical plane waves, $j_{l}$ is a spherical Bessel function, and $Y_{L}$ is a spherical harmonic. Moreover, $\psi_{\alpha}^{\text {ext }}$ can be obtained from the external electric field $\mathbf{E}^{\text {ext }}$ as ${ }^{39}$

$$
\psi_{\alpha}^{\mathrm{ext}}=\left[\begin{array}{c}
\psi_{\alpha}^{M, \mathrm{ext}} \\
\psi_{\alpha}^{E, \mathrm{ext}}
\end{array}\right]=\frac{1}{L_{\alpha}^{2}}\left[\begin{array}{c}
\mathbf{L}_{\alpha} \cdot \mathbf{E}^{\mathrm{ext}} \\
\frac{i}{k \epsilon_{j} \mu_{j}}\left(\mathbf{L}_{\alpha} \times \boldsymbol{\nabla}\right) \cdot \mathbf{E}^{\mathrm{ext}}
\end{array}\right] .
$$

Now let us consider a cluster of dielectric objects labeled by nearby positions $\mathbf{r}_{\alpha}$ and surrounded by a homogeneous medium $j=0$. This is represented in Fig. 1 for two objects $\alpha$ and $\beta$ interacting with each other as well as with an external electron, which provides the external field $\psi^{\text {ext }}$. The total electric field can be separated as $\mathbf{E}=\mathbf{E}^{\text {ext }}+\mathbf{E}^{\text {ind }}$, where the second term (i.e., the induced field) finds its sources in the charges and currents induced in the dielectric objects, and therefore, it has to be comprised of outgoing spherical waves centered at the latter:

$$
\psi^{\text {ind }}(\mathbf{r})=\sum_{\alpha} \psi_{\alpha}^{\text {ind }}(\mathbf{r})
$$

where

$$
\psi_{\alpha}^{\text {ind }}(\mathbf{r})=\sum_{L} h_{L}^{(+)}\left[k_{0}\left(\mathbf{r}-\mathbf{r}_{\alpha}\right)\right] \psi_{\alpha, L}^{\text {ind }},
$$

$h_{L}^{(+)}(\mathbf{u})=i^{l} h_{l}^{(+)}(|\mathbf{u}|) Y_{L}(\hat{\mathbf{u}})$, and $h_{L}^{(+)}$is a spherical Hankel function. ${ }^{41}$ This expression is valid for $\mathbf{r}$ outside a sphere

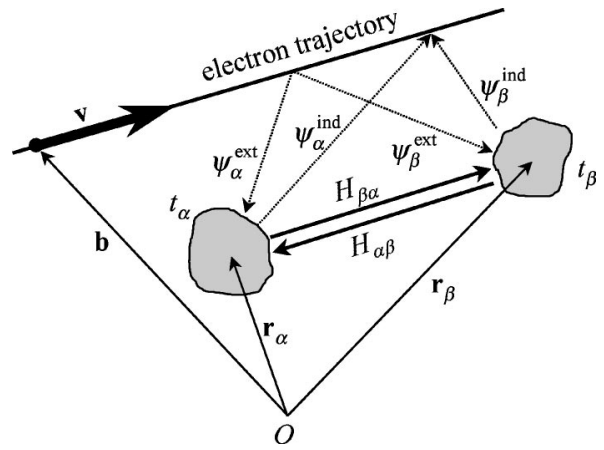

FIG. 1. Schematic representation of the multiple-scattering approach to the study of the interaction of an external electron with a cluster of dielectric objects. Only two objects, $\alpha$ and $\beta$, have been drawn for simplicity. They are located near the positions $\mathbf{r}_{\alpha}$ and $\mathbf{r}_{\beta}$ with respect to the origin $O$. The external field set up by the electron, whose trajectory is defined by $\mathbf{r}=\mathbf{b}+\mathbf{v} t$, can be expanded in terms of scalar functions $\psi^{\text {ext }}$ around $\mathbf{r}_{\alpha}$ and $\mathbf{r}_{\beta}$. Its interaction with these objects is described by scattering matrices $t_{\alpha}$ and $t_{\beta}$. The self-consistently scattered (induced) field $\psi^{\text {ind }}$ is propagated between centers by means of matrices $H$. The induced field acts back on the electron producing stopping.

centered at $\mathbf{r}_{\alpha}$ and fully containing object $\alpha$, and, in particular, at the position of the trajectory followed by the external electron considered in Fig. 1, on which the induced field acts to produce energy loss, as discussed in Sec. III.

Part of the induced field results from single scattering (ss) of the external field at the objects of the cluster, that is, the field obtained by neglecting subsequent multiple-scattering events. In the linear-response approximation, this contribution is proportional to the external field, and the coefficients of proportionality form the so-called scattering matrix $t_{\alpha}$, implicitly defined by

$$
\psi_{\alpha, L}^{\mathrm{ss}}=\sum_{L^{\prime}} t_{\alpha, L L^{\prime}} \psi_{\alpha, L^{\prime}}^{\mathrm{ext}}
$$

$t_{\alpha}$ can be obtained by solving Maxwell's equations in the presence of an object $\alpha$. If the latter is spherically symmetric, one has $t_{\alpha, L L^{\prime}}=t_{\alpha, l} \delta_{L L^{\prime}}$, and magnetic and electric components are not mixed during scattering. ${ }^{39}$ In particular, analytical expressions are obtained for both homogeneous spheres and coated spheres in terms of the local frequencydependent dielectric functions of the materials of which they are made. ${ }^{19,40}$

The self-consistent induced field $\psi_{\alpha}^{\text {ind }}$ is the sum of $\psi^{\text {ss }}$ plus the result of the free propagation of $\psi_{\beta}^{\text {ind }}$ from each object $\beta \neq \alpha$ followed by scattering at $\alpha$ (see Fig. 1). This leads to the self-consistent relation ${ }^{42}$

$$
\widetilde{\psi}_{\alpha}^{\text {ind }}=\widetilde{\psi}_{\alpha}^{\text {ss }}+t_{\alpha} \sum_{\beta \neq \alpha} H_{\alpha \beta} \widetilde{\psi}_{\beta}^{\text {ind }}
$$

where matrix notation has been adopted (i.e., the vector $\widetilde{\psi}_{\alpha}^{\text {ss(ind) }}$ has components $\psi_{\alpha, L}^{\text {ss(ind) }}$ and the matrix $t_{\alpha}$ has components $\left.t_{\alpha, L L^{\prime}}\right)$. The matrix

$$
H_{\alpha \beta}=R_{\alpha \beta}^{-1} T_{\alpha \beta}^{z} G_{\alpha \beta}^{z} R_{\alpha \beta}
$$


TABLE I. Explicit expressions of $(\mathrm{V} / c)^{l+1} \gamma^{l} \sqrt{\pi /(2 l+1)} A_{L}^{+}$for $m \geqslant 0$ and $l \leqslant 4$. Notice that these are polynomials of order $l-m$ in $\gamma$. In addition, $A_{l,-m}^{+}=(-1)^{m} A_{L}^{+}$.

\begin{tabular}{lccccc}
\hline \hline & $m=0$ & $m=1$ & $m=2$ & $m=3$ & $m=4$ \\
\hline$l=0$ & 1 & & & \\
$l=1$ & $i \gamma$ & $-1 / \sqrt{2}$ & & \\
$l=2$ & $-1 / 2-\gamma^{2}$ & $-i \sqrt{\frac{3}{2}} \gamma$ & $\sqrt{\frac{3}{8}}$ & \\
$l=3$ & $-i \gamma\left(\frac{3}{2}+\gamma^{2}\right)$ & $\sqrt{3}\left(\frac{1}{4}+\gamma^{2}\right)$ & $i \sqrt{\frac{15}{8}} \gamma$ & $-\sqrt{\frac{5}{16}}$ & \\
$l=4$ & $\frac{3}{8}+3 \gamma^{2}+\gamma^{4}$ & $i \sqrt{5} \gamma\left(\frac{3}{4}+\gamma^{2}\right)$ & $-\sqrt{\frac{5}{32}}\left(1+6 \gamma^{2}\right)$ & $-i \sqrt{\frac{35}{16}} \gamma$ & $\sqrt{\frac{35}{128}}$ \\
\hline \hline
\end{tabular}

accounts for the aforementioned propagation, conveniently separated into rotation matrices $R_{\alpha \beta},{ }^{43,41}$ translation of spherical harmonics along the $z$ axis a distance $d_{\alpha \beta},{ }^{44}$

$$
\begin{aligned}
G_{\alpha \beta, L L^{\prime}}^{z}= & \delta_{m m^{\prime}} \sqrt{4 \pi} \sum_{l^{\prime \prime}=\left|l-l^{\prime}\right|}^{l+l^{\prime}} \sqrt{2 l^{\prime \prime}+1} i^{l^{\prime \prime}} h_{l^{\prime \prime}}^{(+)}\left(k_{0} d_{\alpha \beta}\right) \\
& \times \int d \Omega Y_{l^{\prime} m}^{*}(\Omega) Y_{l^{\prime \prime} 0}(\Omega) Y_{l m}(\Omega)
\end{aligned}
$$

and translation of the origin of multipoles, ${ }^{40}$

$$
\begin{aligned}
T_{\alpha \beta, L L^{\prime}}^{z}= & {\left[\begin{array}{cc}
\delta_{L L^{\prime}} & 0 \\
0 & \delta_{L L^{\prime}}
\end{array}\right] } \\
& +\delta_{m m^{\prime}} k_{0} d_{\alpha \beta}\left[\begin{array}{cc}
p_{l l^{\prime} m} & q_{l l^{\prime} m} \sqrt{\epsilon_{0} \mu_{0}} \\
\frac{-q_{l l^{\prime} m}}{\sqrt{\epsilon_{0} \mu_{0}}} & p_{l l^{\prime} m}
\end{array}\right],
\end{aligned}
$$

where

$$
\begin{gathered}
q_{l l^{\prime} m}=\frac{m \delta_{l, l^{\prime}}}{l(l+1)}, \\
p_{l l^{\prime} m}=\delta_{l+1, l^{\prime}} D_{l+1, m}-\delta_{l-1, l^{\prime}} D_{L},
\end{gathered}
$$

and

$$
D_{L}=\frac{i}{l} \sqrt{\frac{(l+m)(l-m)}{(2 l-1)(2 l+1)}} .
$$

Notice that, unlike $G_{\alpha \beta}^{z}, T_{\alpha \beta}^{z}$ mixes electron and magnetic components via off-diagonal terms $q$ [see Eq. (9)].

The induced electric field is then obtained in the presence of the cluster by solving Eq. (6) with the input of the ss field expressed in terms of the external field by means of Eqs. (2) and (5), and by inserting the resulting coefficients $\psi_{\alpha, L}^{\text {ind }}$ into Eq. (4), and this in turn into Eqs. (3) and (1). It can be shown ${ }^{40}$ that the factorization of Eq. (7) and the sparse character of the operators given in Eqs. (8) and (9) permit one to solve Eq. (6) in a time proportional to $\approx N^{2}\left(l_{\max }+1\right)^{3}$, where $N$ is the number of objects in the cluster and $l_{\max }$ is the maximum value of the orbital angular momentum numbers $l$ under consideration [i.e., $\widetilde{\psi}_{\alpha}^{\text {ind }}$ is actually approximated by a finite vector of dimension $\left(l_{\max }+1\right)^{2}$ for each component, electric and magnetic]. Convergence has been achieved for $l_{\max }=12$ in most of the examples offered below.

\section{APPLICATION TO ELECTRON-ENERGY-LOSS SPECTROSCOPY}

Expressions for the electron-energy-loss probability will be derived in this section for a fast electron moving near a cluster of dielectric objects with constant velocity $\mathbf{v}$. The cluster will be assumed to be surrounded by vacuum (i.e., $\epsilon_{0}=\mu_{0}=1$ and $\left.k_{0}=k=\omega / c\right)$. The electron trajectory will be taken to be fully contained in the vacuum side and defined by the equation $\mathbf{r}=\mathbf{b}+\mathbf{v} t$ (see Fig. 1). For simplicity, the velocity will be first considered to be directed along the $z$ direction, and results for arbitrary directions will be expressed in terms of those obtained for this particular case.

The external electric field set up by the fast electron can be written in terms of multipole components around a given cluster scattering center $\mathbf{r}_{\alpha}$ as ${ }^{19}$

$$
\mathbf{E}_{\alpha}^{\mathrm{ext}}(\mathbf{r})=\left(\nabla-\frac{i k \mathbf{v}}{c}\right) \sum_{L} i^{-l} j_{L}\left[k\left(\overline{\mathbf{r}}-\overline{\mathbf{r}}_{\alpha}\right)\right] \phi_{\alpha, L},
$$

where

$$
\phi_{\alpha, L}=4 \pi k \frac{A_{L}^{+}}{\omega} K_{m}\left[\frac{\omega b_{\alpha}}{\mathrm{v} \gamma}\right] e^{-i m \varphi_{\alpha}-i \omega z_{\alpha} / \mathrm{v}},
$$

$A_{L}^{+}$is an analytical coefficient that depends exclusively on $\mathrm{V} / c$, as shown in Eq. (A1) of the Appendix; $K_{m}$ is the modified Bessel function; $\gamma=1 / \sqrt{1-(\mathrm{V} / c)^{2}}$; and $\left(b_{\alpha}, \varphi_{\alpha}, z_{\alpha}\right)$ are the cylindrical coordinates of $\mathbf{b}-\mathbf{r}_{\alpha}$. The coefficients of the multipole expansion of the scalar functions can be obtained by substituting Eq. (10) for $\mathbf{E}$ in Eq. (2), leading to the analytical expression ${ }^{19}$

$$
\left[\begin{array}{c}
\psi_{\alpha, L}^{M, \mathrm{ext}} \\
\psi_{\alpha, L}^{E, \mathrm{ext}}
\end{array}\right]=\frac{-2 \pi i^{1-l} k}{l(l+1) c}\left[\begin{array}{c}
2 m A_{L}^{+} \mathrm{v} / c \\
B_{L} / \gamma
\end{array}\right] K_{m}\left[\frac{\omega b_{\alpha}}{\mathrm{v} \gamma}\right] e^{-i m \varphi_{\alpha}-i \omega z_{\alpha} / \mathrm{v}},
$$

where $B_{L}$ is a combination of the coefficients $A_{L}^{+}$, as shown in Eq. (A2) of the Appendix. Tables I and II offer analytical expressions for the first values of these coefficients.

The energy-loss probability can be derived from the retarding force produced by the induced electric field $\mathbf{E}^{\text {ind }}$ acting back on the electron as ${ }^{37}$

$$
\Delta E^{\text {loss }}=\int d t \mathbf{v} \cdot \mathbf{E}^{\text {ind }}\left(\mathbf{r}_{t}, t\right)=\int_{0}^{\infty} \omega d \omega \Gamma^{\operatorname{loss}}(\omega),
$$

where 
TABLE II. Explicit expressions of $(\mathrm{V} / c)^{l+1} \gamma^{l} \sqrt{\pi /(2 l+1)} B_{L}$ for $m \geqslant 0$ and $l \leqslant 4$. Notice that these are polynomials of order $l-|| m|-1|$ in $\gamma$. In addition, $B_{l,-m}=(-1)^{m} B_{L}$.

\begin{tabular}{lccccc}
\hline \hline & $m=0$ & $m=1$ & $m=2$ & $m=3$ & $m=4$ \\
\hline$l=0$ & 0 & $-i \sqrt{2} \gamma$ & & \\
$l=1$ & -2 & $\sqrt{6}\left(1+\gamma^{2}\right)$ & $i \sqrt{6} \gamma$ & \\
$l=2$ & $-6 i \gamma$ & $i \sqrt{3} \gamma\left(\frac{11}{2}+2 \gamma^{2}\right)$ & $-\sqrt{\frac{15}{2}}\left(1+2 \gamma^{2}\right)$ & $-i \sqrt{\frac{45}{4}} \gamma$ & \\
$l=3$ & $3+12 \gamma^{2}$ & $-\sqrt{\frac{5}{4}}\left(3+21 \gamma^{2}+4 \gamma^{4}\right)$ & $-i \sqrt{10} \gamma\left(4+3 \gamma^{2}\right)$ & $\sqrt{\frac{35}{4}}\left(1+3 \gamma^{2}\right)$ & $i \sqrt{\frac{35}{2}} \gamma$ \\
$l=4$ & $5 i \gamma\left(3+4 \gamma^{2}\right)$ & & \\
\hline \hline
\end{tabular}

$$
\Gamma^{\operatorname{loss}}(\omega)=\frac{1}{\pi \omega} \int d t \operatorname{Re}\left\{e^{-i \omega t} \mathbf{v} \cdot \mathbf{E}^{\text {ind }}\left(\mathbf{r}_{t}, \omega\right)\right\}
$$

is the so-called loss probability from which loss spectra are calculated here. The latter can be decomposed into contributions coming from the self-consistent field scattered by each component of the cluster $\alpha$ and its magnetic and electric multipoles as

$$
\Gamma^{\text {loss }}=\sum_{L, \alpha} \Gamma_{L, \alpha}^{M, \text { loss }}+\Gamma_{L, \alpha}^{E, \text { loss }}
$$

These terms can be now calculated analytically by inserting the coefficients $\psi_{\alpha, L}^{\text {ind }}$ obtained from Eq. (6) into Eq. (4), and this in turn into Eqs. (1) and (12). One finds ${ }^{19}$

$\Gamma_{\alpha, L}^{M, \operatorname{loss}}(\omega)=\frac{m \mathrm{v}}{\pi \omega^{2}} K_{m}\left[\frac{\omega b_{\alpha}}{\mathrm{v} \gamma}\right] \operatorname{Re}\left\{\left(A_{L}^{+}\right)^{*} e^{\left.i m \varphi_{\alpha}+i \omega z_{\alpha} / \mathrm{v}_{i}^{l} \psi_{\alpha, L}^{M, \text { ind }}\right\}}\right.$

and

$$
\Gamma_{\alpha, L}^{E, \text { loss }}(\omega)=\frac{c}{2 \pi \omega^{2} \gamma} K_{m}\left[\frac{\omega b_{\alpha}}{\mathrm{v} \gamma}\right] \operatorname{Re}\left\{B_{L}^{*} e^{i m \varphi_{\alpha}+i \omega z_{\alpha} / v_{i}^{l}} \psi_{\alpha, L}^{E, \text { ind }}\right\}
$$

For an isolated object, the self-consistent field is given by Eq. (5). Then, using Eq. (11), Eq. (13) reduces to

$$
\begin{aligned}
\Gamma^{\operatorname{loss}}(\omega)= & \frac{1}{c \omega} \sum_{L L^{\prime}} K_{m}\left(\frac{\omega b}{\mathrm{v} \gamma}\right) K_{m^{\prime}}\left(\frac{\omega b}{\mathrm{v} \gamma}\right) \\
& \times \operatorname{Im}\left\{\frac{i^{l-l^{\prime}}}{l^{\prime}\left(l^{\prime}+1\right)} e^{i\left(m-m^{\prime}\right) \varphi}\left[\frac{2 m \mathrm{v}\left(A_{L}^{+}\right) *}{c} \frac{B_{L}^{*}}{\gamma}\right]\right. \\
& \left.\times\left[\begin{array}{cc}
t_{L L^{\prime}}^{M M} & t_{L L^{\prime}}^{M E} \\
t_{L L^{\prime}}^{E M} & t_{L L^{\prime}}^{E E}
\end{array}\right]\left[\begin{array}{c}
\frac{2 m^{\prime} \mathrm{\vee} A_{L^{\prime}}^{+}}{c} \\
\frac{B_{L^{\prime}}}{\gamma}
\end{array}\right]\right\},
\end{aligned}
$$

where the $t$ matrix has been separated into four pieces connecting electric and magnetic components, and the subindex $\alpha$ has been dropped. If the object is spherically symmetric, the analytical result for the energy-loss probability for an electron passing at a distance $b$ from its center is given by $^{37,19}$

$$
\Gamma^{\operatorname{loss}}(\omega)=\frac{1}{c \omega} \sum_{L} K_{m}^{2}\left(\frac{\omega b}{\mathrm{v} \gamma}\right)\left[C_{L}^{M} \operatorname{Im}\left\{t_{l}^{M}\right\}+C_{L}^{E} \operatorname{Im}\left\{t_{l}^{E}\right\}\right],
$$

where $t_{l}^{M}$ and $t_{l}^{E}$ are the magnetic and electric parts of the scattering matrix (notice that no mixing of magnetic and electric modes occurs in spherically symmetric objects), the positive coefficients

$$
C_{L}^{M}=\frac{1}{l(l+1)}\left|\frac{2 m \mathrm{v}}{c} A_{L}^{+}\right|^{2}
$$

and

$$
C_{L}^{E}=\frac{1}{l(l+1)}\left|\frac{1}{\gamma} B_{L}\right|^{2}
$$

depend exclusively on the ratio $\mathrm{V} / c$, and $A_{L}^{+}$and $B_{L}$ are defined in Appendix A (see also Tables I and II).

A detailed discussion of the energy loss in the presence of isolated homogeneous spheres has been given elsewhere. ${ }^{19}$ Here some results are included for the sake of comparison in the case of homogeneous Al spheres (the lower curve in Fig. 2 ), the sum of contributions coming from different spheres without including their mutual interaction in what represents the single scattering (ss) approach discussed in Sec. II (e.g., see the broken curves in Fig. 3), and isolated Si spheres coated with $\mathrm{SiO}_{2}$ (the upper part of Fig. 5).

When the velocity is oriented along an arbitrary direction, the above expressions can be rotated with the help of rotation matrices. Indeed, the external scalar functions are given by $R_{\mathbf{v}}^{-1} \psi^{\text {ext }}$, where $\psi^{\text {ext }}$ are the scalar functions corresponding to the case where the velocity is oriented along the $z$ direction [i.e., Eq. (11)], and $R_{\mathbf{v}}$ is the rotation matrix that brings the velocity vector $\mathbf{v}$ onto the $z$ axis. This rotation is defined by the Euler angles ${ }^{41}\left(0, \theta_{\mathbf{v}}, \pi-\varphi_{\mathbf{v}}\right)$, where $\left(\theta_{\mathbf{v}}, \varphi_{\mathbf{v}}\right)$ are the polar angles of $\mathbf{v}$. In addition, Eqs. (14) and (15) remain valid if one substitutes $\psi^{\text {ind }}$ with $R_{\mathbf{v}} \psi^{\text {ind }}$. Of course, the cluster coordinates $\mathbf{r}_{\alpha}$ and the electron-impact parameter $\mathbf{b}$ have to be rotated as well, using the representation of these rotation matrices in three-dimensional space. ${ }^{41}$

\section{RESULTS AND DISCUSSION}

The present formalism has been applied to calculate EELS for electrons moving near clusters of different sizes formed by spheres. The spheres are made of $\mathrm{Al}, \mathrm{SiO}_{2}$, and $\mathrm{Si}$. The scattering matrices of the spheres have been obtained using analytical expressions in terms of the frequencydependent dielectric functions of these materials (see Sec. 


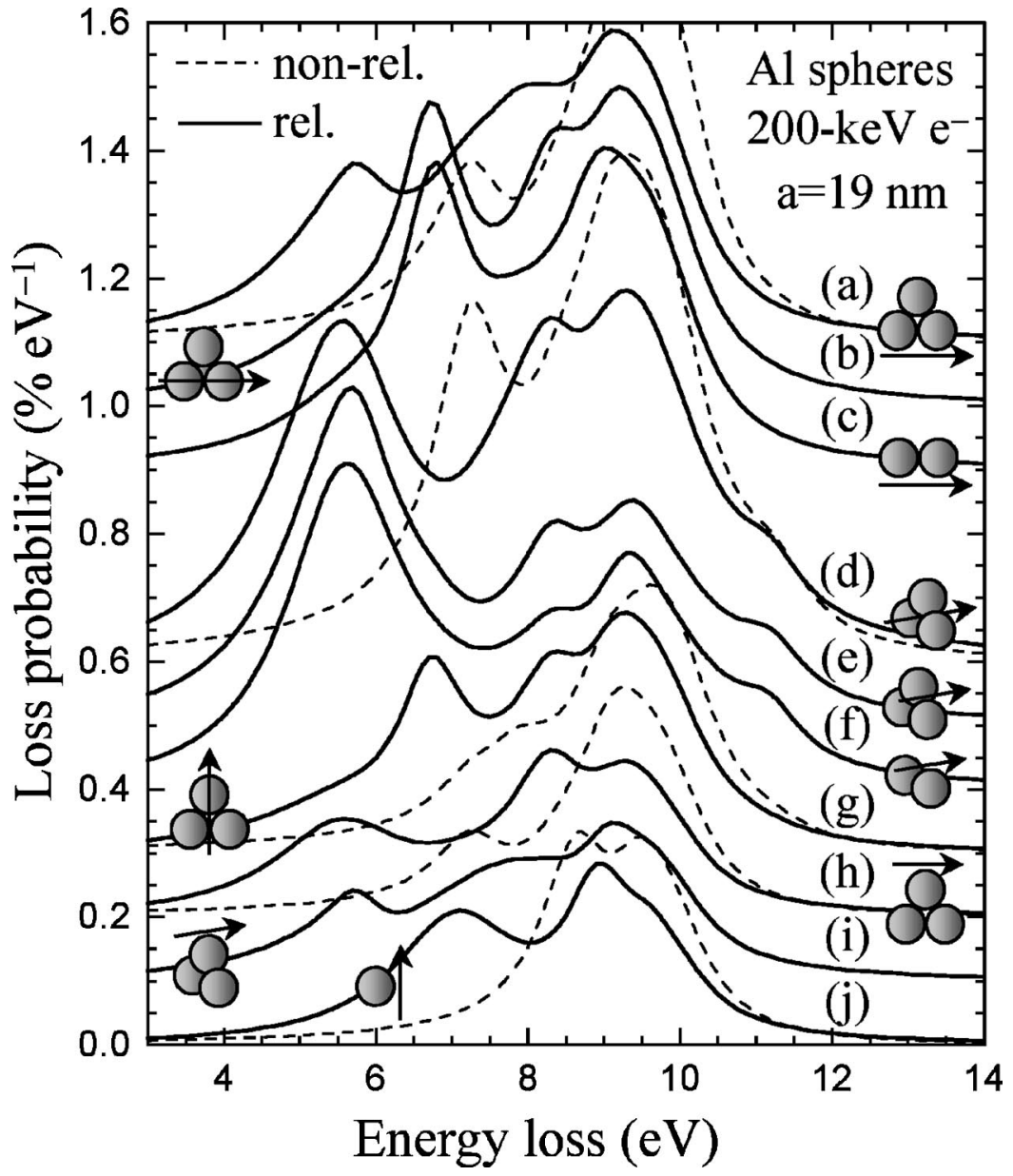

FIG. 2. Energy-loss probability per energy range for a $200-\mathrm{keV}$ electron (i.e., $\mathrm{v}=0.695 c$ $=95.3$ a.u.) moving near clusters of $1-3 \mathrm{Al}$ spheres along trajectories shown in the insets. Fully relativistic results (solid curves) are compared with nonrelativistic ones (broken curves, only for some of the geometries). The radius of the spheres is $a=19 \mathrm{~nm}$, the separation between sphere surfaces is $2 \mathrm{~nm}$, and the distance between the electron trajectory and the sphere surfaces is $4.1 \mathrm{~nm}$ for all clusters under consideration. The electron trajectory has been chosen to be directed either parallel or perpendicular to the plane formed by the sphere centers. Consecutive curves have been shifted $0.1 \% \mathrm{eV}^{-1}$ upwards to improve readability.
II B of Ref. 40 for explicit expressions). In particular, the response of $\mathrm{Al}$ has been approximated by a Drude dielectric function with bulk plasma energy $\omega_{p}=15 \mathrm{eV}$ and damping $\eta=1.06 \mathrm{eV}$. The dielectric functions of $\mathrm{Si}$ and $\mathrm{SiO}_{2}$ have been taken from optical data. ${ }^{45}$

The results obtained with the boundary element method for systems of aligned spheres ${ }^{37}$ are reproduced using the present formalism. These two methods are somehow complementary to each other, since the former allows objects of arbitrary shape to be studied and the latter permits one to combine a large number of those objects located in arbitrary positions.

The effects of retardation and interaction among different components of a cluster have been analyzed in Fig. 2 for an isolated sphere [Fig. 2(j)], a two-sphere cluster [Figs. 2(c) and $2(\mathrm{f})]$, and a cluster of three equidistant spheres (the rest of the figure). The sphere radius is $a=19 \mathrm{~nm}$, the distance between sphere surfaces is $2 \mathrm{~nm}$, and the distance of closest approach of the electron relative to the sphere surfaces is 4.1 $\mathrm{nm}$ in all cases. Fully relativistic results (solid curves) exhibit remarkable differences with respect to nonrelativistic results (broken curves). The latter are numerically obtained by taking unrealistic large values of the speed of light, and convergence in self-consistently solving Eq. (6) is generally poorer than in the relativistic case. The effect of retardation is translated into a shift of the modes towards lower energies, in agreement with previous results. ${ }^{37,19}$ In Figs. 2(h)-2(j), the spectrum is mainly influenced by the sphere nearest to the trajectory, and one can observe the emergence of new low-energy peaks in the three-sphere cluster [Figs. 2(h) and 2(i)] as compared with the isolated sphere [Fig. 2(j)]. Moreover, different trajectories excite the modes of the cluster with different probabilities, leading to different spectral shapes as can be seen in Fig. 2(g) for the same three-sphere cluster, with a peak showing up at $6.7 \mathrm{eV}$, where there was a valley in Fig. 2(h). The spectrum for a trajectory perpendicular to a two-sphere cluster [Fig. 2(f)] does not change much when adding a new sphere on the far side with respect to the trajectory, as shown in Fig. 2(e). However, the strength of the peaks is changed if the sphere is added in a position such that the trajectory passes by the center of the cluster [Fig. 2(d)]. A similar analysis can be carried out by observing Figs. 2(a)-2(c). In particular, by comparison with Fig. 2(b), the low-energy peak at $5.6 \mathrm{eV}$ in Fig. 2(a) seems to be due to the interaction of the two spheres closer to the trajectory with the sphere opposite to it.

This latter effect has been further explored in Fig. 3, where two identical Al spheres are placed near the electron trajectory and the role of a third sphere of $\mathrm{Al}$ (the left part of the figure) or $\mathrm{SiO}_{2}$ (the right part) is illustrated as a function of its radius $a$ (see labels and inset in the figure). In this case, the broken curves stand for the result of neglecting the interaction between spheres (i.e., the single-scattering approach), and they show that the direct contribution of the third sphere can only be observed in the low-energy end of the spectra and just when it is made of Al. Therefore, the bulk of the effect of adding the third sphere comes through multiple scattering (solid curves). The interaction of the third sphere 


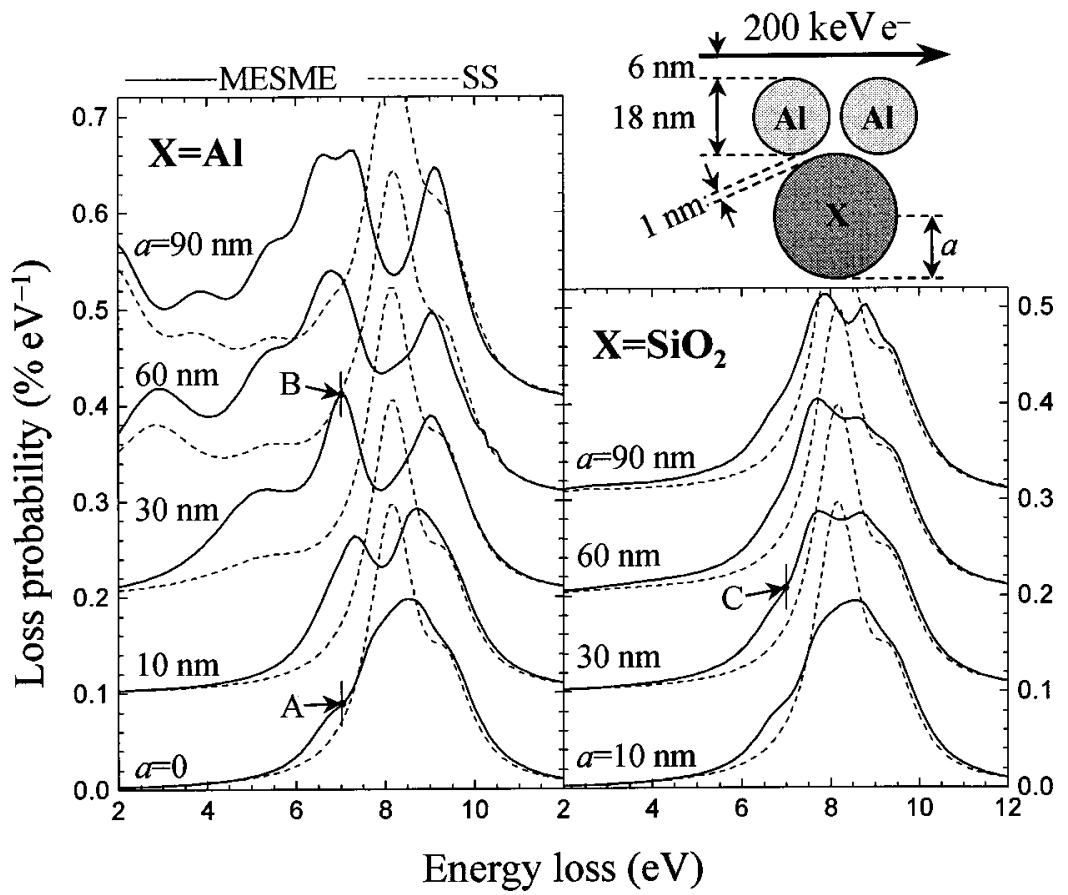

with the rest of the cluster increases with its radius, and it is stronger in the case of $\mathrm{Al}$ as compared with $\mathrm{SiO}_{2}$.

Contour maps of the square of the induced electric field strength for an energy loss $\omega=7 \mathrm{eV}$ are shown in Fig. 4 for clusters corresponding to points $A, C$, and $B$ of Fig. 3 [Figs. 4(a)-4(c), respectively]. The $\mathrm{SiO}_{2}$ sphere of radius $a=30$ $\mathrm{nm}$ considered in Fig. 4(b) does not produce significant changes in the distribution of the induced field as compared with the two-Al-sphere cluster represented in Fig. 4(a). However, when an Al sphere is added [Fig. 4(c)], the electric field suffers large fluctuations in the region between the three spheres, which are translated into significant changes of the induced field near the electron trajectory, and therefore, into different values of the loss probability (see points $A$ and $B$ in Fig. 3).

Some results for $\mathrm{Si}$ spheres coated with $\mathrm{SiO}_{2}$ are presented in Fig. 5. The sphere radius is $a=18 \mathrm{~nm}$ in all cases and the radius of the inner $\mathrm{Si}$ core varies from $b=0$ (pure $\mathrm{SiO}_{2}$ spheres) to $b=a$ (pure $\mathrm{Si}$ spheres). The exciton mode of $\mathrm{SiO}_{2}$ shows up at around $10.5 \mathrm{eV}$ when $b<a$. The interaction for energies below this peak is very weak in the pure $\mathrm{SiO}_{2}$ case, and this responds to the fact that the imaginary part of the dielectric function is very small, and hence, most of the losses are due to radiation emission. ${ }^{37,19}$ Important changes occur in the strength of the spectral features when one compares pure $\mathrm{Si}$ spheres and $\mathrm{Si}$ spheres coated with a layer of only $2 \mathrm{~nm}$ of $\mathrm{SiO}_{2}$, though the energy of those features does not change significantly. Figure 5(a) shows results for isolated spheres (multiplied by a factor of three), whereas Fig. 5(b) corresponds to a three-sphere cluster, as shown in the inset. As in previous examples, the mutual interaction among spheres produces splitting and displacement of the features of the energy-loss spectra.

Larger clusters have been considered in Figs. 6-10. In particular, Fig. 6 illustrates how the interaction among the spheres of octahedral clusters of $\mathrm{Al}$ can lead to dramatic effects in EELS. Results for noninteracting spheres (broken curves) are very different from those arising from full mul-
FIG. 3. Energy-loss probability per energy range for a $200-\mathrm{keV}$ electron moving near a cluster of three spheres, as shown in the inset. The trajectory and the sphere centers lie all in the same plane. The two upper Al spheres are identical. The lower sphere is taken to be made of $\mathrm{Al}$ $\left(\mathrm{SiO}_{2}\right)$ on the left (right) part of the figure, and different values of its radius $a$ have been considered, as indicated by labels. Full multiple elasticscattering results (MESME) are shown by solid curves, whereas single-scattering results (ss), standing for noninteracting spheres, are shown by broken curves. Consecutive curves have been shifted $0.1 \% \mathrm{eV}^{-1}$ upwards to improve readability.

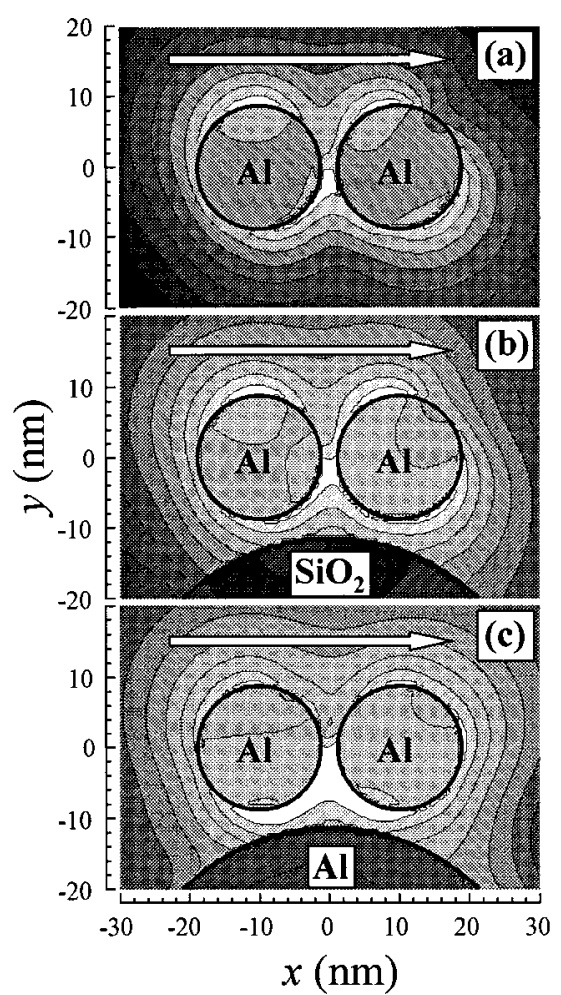

FIG. 4. Spatial distribution of the square of the induced electricfield strength corresponding to an energy loss of $7 \mathrm{eV}$ for the same geometry and parameters as in Fig. 3. The plane of representation contains the trajectory and the sphere centers. (a) stands for two isolated $\mathrm{Al}$ spheres (see point $A$ in Fig. 3). The radius of the lower sphere is $a=30 \mathrm{~nm}$ in (b) and (c), and it is made of $\mathrm{SiO}_{2}$ and $\mathrm{Al}$, respectively (see points $C$ and $B$ in Fig. 3). The white arrows indicate the direction and position of the electron trajectory. The difference between consecutive contour curves represents a constant factor of $10^{-0.2}$ 


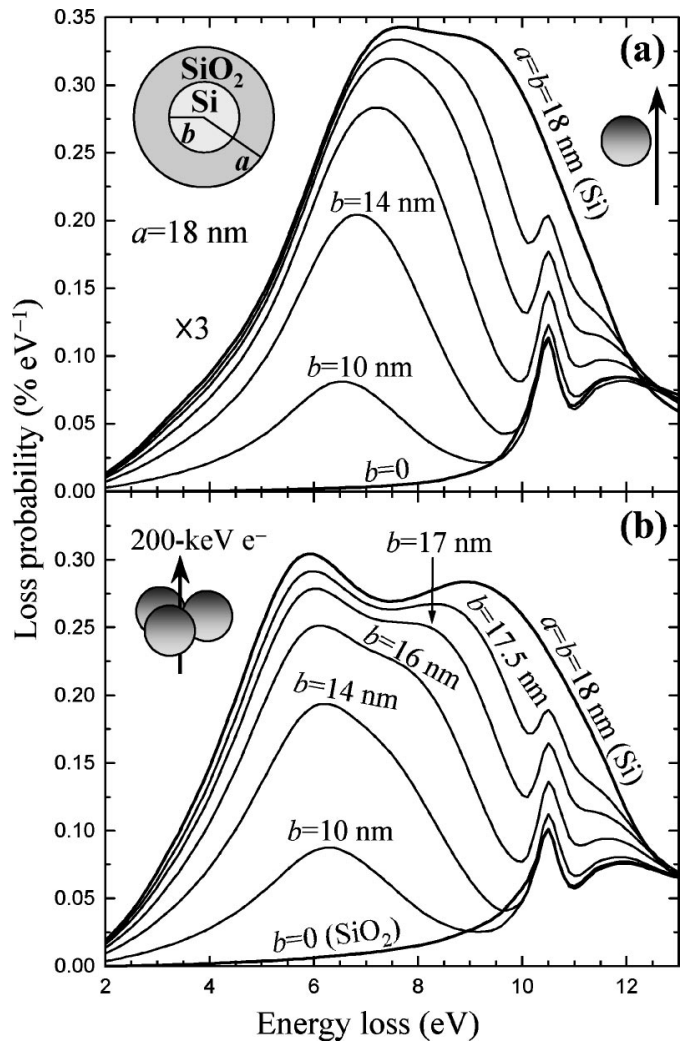

FIG. 5. Energy-loss probability per energy range experienced by a 200-keV electron moving near (a) an isolated coated sphere (multiplied by a factor of 3) and (b) a cluster formed by three equidistant spheres. The spheres have an inner spherical core of radius $b$ made of $\mathrm{Si}$, and they are coated with $\mathrm{SiO}_{2}$. Different values of $b$ have been considered, as shown by labels. The external sphere radius is $a=18 \mathrm{~nm}$, the bond distance in the three-sphere cluster is $d=40$ $\mathrm{nm}$, and the distance from the trajectory to the sphere centers is $d / \sqrt{3} \approx 23.1 \mathrm{~nm}$ in all cases. The trajectory in the lower figure is perpendicular to the plane determined by the sphere centers (see inset).

tiple scattering (solid curves) in the case of touching spheres [Figs. 6(a)-6(e)]. For nontouching spheres with constant bond distance, the interaction between spheres decreases with decreasing radius [Figs. 6(e)-6(h)].

Figure 7 shows energy-loss spectra for an electron crossing a planar triangular mesh of $\mathrm{Al}$ spheres of different radius $a$ and bond distances $d$, as shown in the figure. Only $N$ nearest neighbors with respect to the electron trajectory have been included in the calculation, with $N=3,12$, and 30 , and a value sufficiently large to ensure convergence in the $N$ $\rightarrow \infty$ limit. This value corresponds to $N=198,141$, and 30 in the upper, middle, and lower sets of curves, respectively. $N$ $=30$ shows already convergence for energy losses above 7 $\mathrm{eV}$ in all cases. Dramatic changes in the loss spectra are observed as compared with the non-interacting-spheres description (thick broken curves), especially when the distance between sphere surfaces decreases. Single-scattering results show convergence in the $N \rightarrow \infty$ limit for $N=12$, though $N$ $=3$ deviates less than $5 \%$ from the converged result. Therefore, the interaction with distant spheres is crucial and is strongly mediated by multiple-scattering effects, that is, by propagation of collective modes throughout the cluster, in

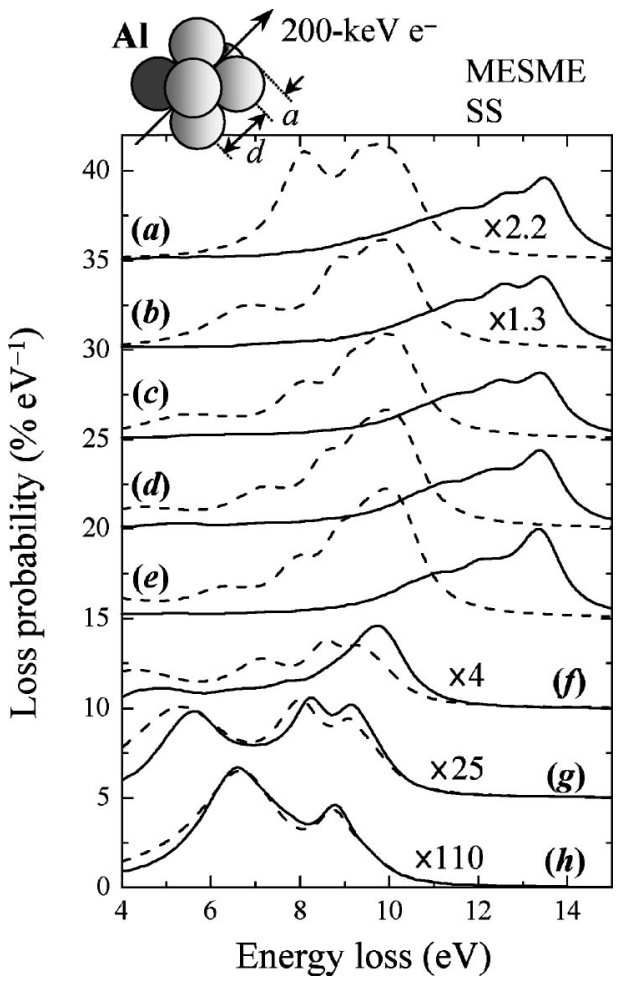

FIG. 6. Electron-energy-loss probability per energy range for a 200-keV electron passing by the center of an octahedral cluster of Al spheres. Single-scattering results (shown by dashed curves, which are equal to noninteracting spheres) and full multiplescattering results (MESME; solid curves) are shown for clusters of touching spheres with bond distances $d=20,40,60,80$, and 100 $\mathrm{nm}$ in (a)-(e), respectively. (f) - (h) correspond to clusters with a constant bond distance $d=100 \mathrm{~nm}$ and a sphere radius $a=40,30$, and $20 \mathrm{~nm}$, respectively. The electron trajectory is chosen to intersect the centers of two opposite edges of the imaginary octahedron that defines the geometry. Some of the curves have been multiplied by a factor, as shown in the figure, and consecutive curves have been shifted $5 \% \mathrm{eV}^{-1}$ upwards to improve readability.

particular in the low-energy end of the spectra under consideration.

The effect of removing one sphere from a 30 -sphere planar cluster is illustrated in Fig. 8, where the missing sphere is represented in the inset by labels $A-D$. Curve $E$ stands for the result obtained without removing any sphere at all. When one of the nearest spheres with respect to the trajectory is removed (see label $A$ and cross in the inset), the energy-loss spectrum undergoes substantial modifications in the strength of its features, though their position in energy remains nearly intact. This is a further indication that long-range interaction via multiple-scattering effects is dominating the loss spectra. Removing spheres further beyond leads to much smaller changes (see curves $B-D$ ).

Partially disordered photonic lattices have been the subject of recent investigations whose purpose is to study the effect of fabrication misalignments. ${ }^{46}$ The role of disorder in planar lattices of spheres is studied in Fig. 9 for the same 30 -sphere cluster as above by introducing a random displacement in the spheres along the direction normal to the mesh. The maximum displacement is $\pm \Delta z$, and different values of this parameter have been considered, as shown by labels. 


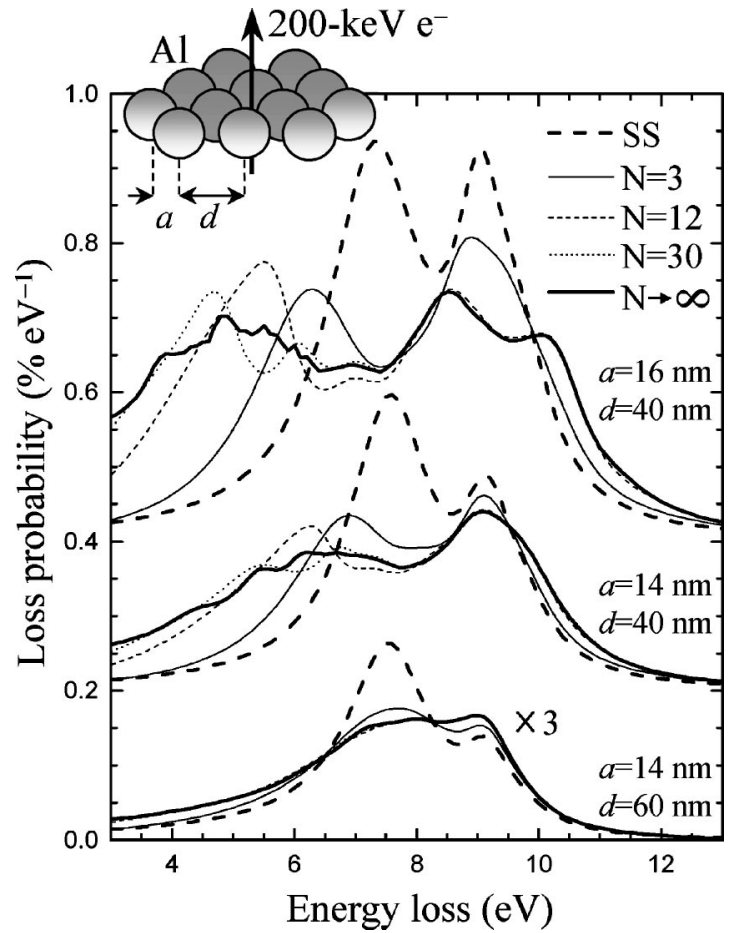

FIG. 7. Electron-energy-loss probability per energy range for a 200-keV electron moving perpendicularly to a plane of Al spheres distributed in a triangular mesh (see the inset). Only the $N$ nearest spheres with respect to the trajectory (from the infinite mesh) have been included in the cluster, and different values of $N$ have been considered from $N=3$ (thin solid curves) up to a large value such that convergence in the $N \rightarrow \infty$ limit is achieved (thick solid curves). Single-scattering results (i.e., those obtained from the noninteracting-spheres picture) are represented by thick dashed curves. Three different sets of values of the sphere radius $a$ and the lattice constant $d$ have been considered (see labels), and curves corresponding to consecutive sets of values have been shifted $0.2 \%$ $\mathrm{eV}^{-1}$ upwards to improve readability. The electron trajectory is chosen to be equidistant from the three nearest spheres.

Ten different clusters have been represented for each value of $\Delta z$. Notice that the role of disorder is negligible for displacements smaller than $10 \%$ of the lattice constant. When $\Delta z$ increases, the different features of the loss spectrum are smeared out.

A tight binding scheme has been used in Fig. 10 for the same 30-sphere cluster. Only spheres separated by a distance smaller than $d_{\max }$ have been allowed to interact. Different values of $d_{\max }$ have been considered, as shown in the figure. The exact result, obtained by taking $d_{\max }=\infty$, is shown as a thick solid curve. When only the interaction between nearest neighbors is included, the loss spectra is well reproduced for losses above $7.5 \mathrm{eV}$. For lower energies, long-range interaction up to a distance of approximately three times the lattice constant is essential in a detailed description of the spectral features.

In the clusters of $\mathrm{Al}$ spheres considered above, the scattering events have a marked electric character. Actually, the results obtained by setting $t_{l}^{M}=0$ are indistinguishable from the exact results on the scale of the figures. This is consistent with previous investigations on the magnitude of magnetic components of the scattering matrix for Drude spheres. ${ }^{19}$ However, this is not the case in $\mathrm{SiO}_{2}$, where magnetic com-

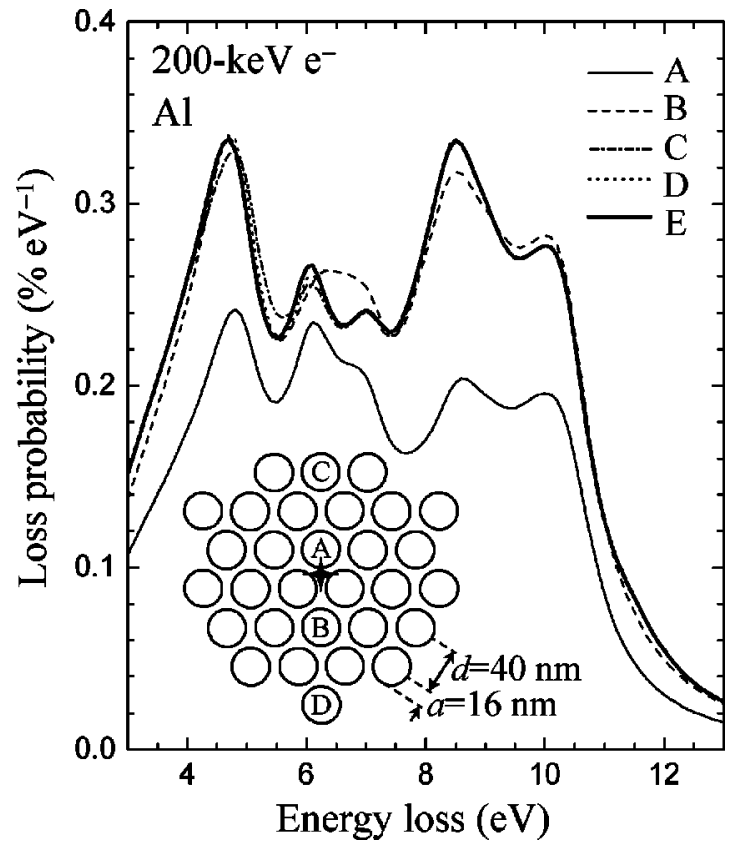

FIG. 8. Electron-energy-loss probability per energy range for a 200-keV electron moving perpendicularly to a plane of $N=30 \mathrm{Al}$ spheres distributed in a finite triangular mesh from which one of the spheres has been removed. The missing sphere corresponds to the labels A-D (see the inset). Curve $E$ stands for the result obtained without removing any sphere at all. A top view of the cluster is shown in the inset, with the trajectory indicated by a cross. The radius of the spheres is $a=16 \mathrm{~nm}$ and the lattice constant is $d$ $=40 \mathrm{~nm}$.

ponents play a significant role. ${ }^{40}$

As a final remark, the fact that no Cherenkov radiation is produced during the interaction of an external electron with an isolated sphere ${ }^{37}$ can be generalized to finite clusters of arbitrary objects when the electron is moving always in vacuum. This can be concluded by examining Eq. (6), symbolically written as

$$
\psi^{\text {ind }}=\frac{1}{1-t H} \psi^{\mathrm{ss}}
$$

which allows one to separate the dependence of the induced field on the electron velocity, fully contained inside $\psi^{\text {ss }}$, from that on the dielectric response, collected inside the scattering matrices $t$. Now, for finite clusters, $t$ and $H$ can be well approximated by finite matrices in Eq. (17), leading, in combination with Eqs. (14) and (15), to expressions for the energy loss that, like Eq. (16) in the case of the isolated sphere, make the Cherenkov condition $(\mathrm{V} / c)^{2} \operatorname{Re}\{\epsilon\}>1$ irrelevant in this case.

\section{CONCLUSIONS}

The theory presented in this work permits one to simulate electron-energy-loss spectra within a fully relativistic approach for electrons passing near clusters of arbitrarily disposed dielectric objects. Relativistic effects are shown to be important in the understanding of energy-loss spectra for object sizes on a nanometer scale, as shown in Fig. 2. Moreover, interaction among objects of the cluster results in dra- 


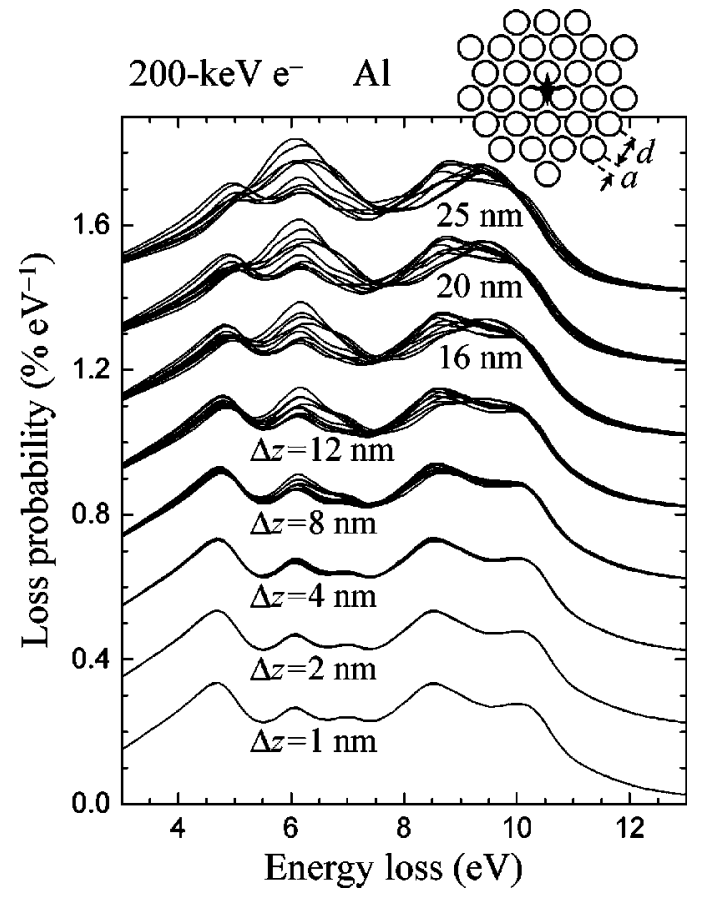

FIG. 9. Electron-energy-loss probability per energy range for a $200-\mathrm{keV}$ electron moving perpendicularly to a plane of $N=30 \mathrm{Al}$ spheres distributed in a finite triangular mesh where partial disorder has been introduced. The spheres have been displaced a random distance along the direction perpendicular to the plane of the mesh with a maximum displacement given by $\pm \Delta z$, as shown by labels. Ten different clusters have been considered for each value of $\Delta z$, and the results of each of them are represented by thin curves. A top view of the cluster is shown in the inset, with the trajectory indicated by a cross. The radius of the spheres is $a=16 \mathrm{~nm}$ and the lattice constant of the planar mesh is $d=40 \mathrm{~nm}$. Curves corresponding to consecutive values of $\Delta z$ have been shifted $0.2 \% \mathrm{eV}^{-1}$ upwards to improve readability.

matic changes of spectral shapes in many cases, as shown in Figs. 2-10. In particular, in Fig. 7, where an electron is considered to be moving perpendicularly to a planar triangular lattice of spheres, the interaction with distant spheres is relevant, and is mainly due to multiple-scattering effects in what can be understood as propagation of the electromagnetic signal throughout the cluster. Actually, long-distance sphere-sphere interaction is relevant in the understanding of energy-loss spectra in clusters, as illustrated in Fig. 10. The interaction among objects of the cluster depends strongly on the size and distance between them, as pointed out in Fig. 6 for octahedral clusters of spheres.

Disorder in a planar lattice has been shown to smear out the main features of the loss spectrum when random displacements of magnitude comparable to the lattice constant are introduced in the direction normal to the plane of the lattice (see Fig. 9). However, the effect of disorder is negligible for displacements below $10 \%$ of the lattice constant in the example considered above.

Radiation scattering cross sections for clusters similar to the ones discussed here show clear similarities with respect to EELS in many cases. ${ }^{40}$

The numerical examples offered above concentrate on clusters of homogeneous spheres and coated spheres. More complicated objects could be incorporated with a similar in-

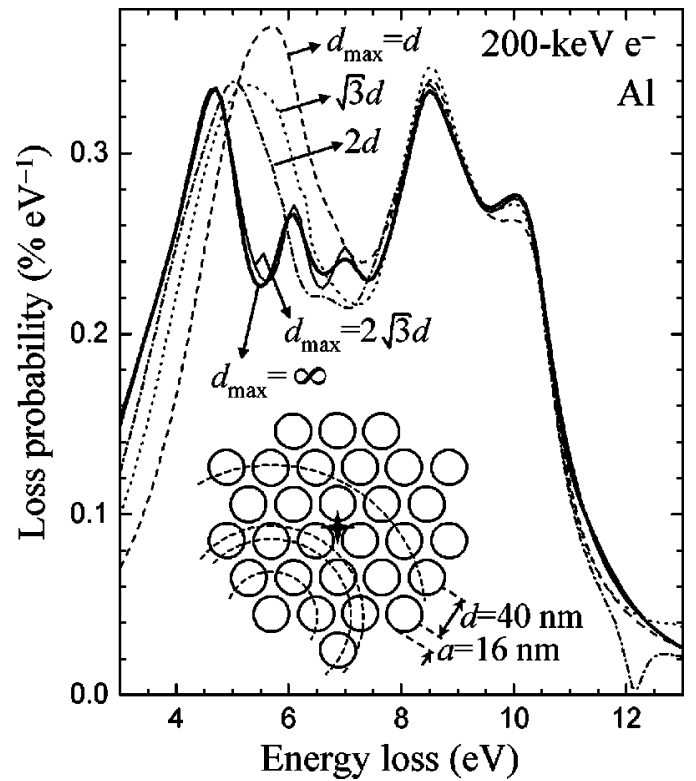

FIG. 10. Electron-energy-loss spectra obtained by using a tightbinding approximation to the multiple-elastic scattering for a 200$\mathrm{keV}$ electron moving perpendicularly to a plane of $N=30 \mathrm{Al}$ spheres distributed in a finite triangular mesh. Only spheres whose bond distance is smaller than $d_{\max }$ have been allowed to interact (see labels in the figure). A top view of the cluster is shown in the inset, with the trajectory indicated by a cross. The different radius of interaction $d_{\max }$ have been represented by circles centered around a sphere located at the edge of the cluster. The radius of the spheres is $a=16 \mathrm{~nm}$, and the lattice constant of the planar mesh is $d=40$ $\mathrm{nm}$.

vestment in computation time, provided one knows the scattering matrices of those objects. Further research in this direction is undergoing, in combination with numerical techniques for solving Maxwell's equations in the presence of arbitrarily shaped dielectrics. ${ }^{37}$

\section{ACKNOWLEDGMENTS}

The author wants to thank M. A. Van Hove and C. S. Fadley for their kind hospitality during his stay in Berkeley, and A. Howie and P. M. Echenique for helpful and enjoyable discussions. Help and support from the University of the Basque Country and the Spanish Ministerio de Educación y Cultura, under Fulbright Grant No. FU-98-22726216, is gratefully acknowledged. Part of this work was supported by the U.S. Department of Energy under contract No. DEAC03-76SF00098.

\section{APPENDIX: COEFFICIENTS $A_{L}^{+}$AND $B_{L}$}

Here analytical expressions for the coefficients $A_{L}^{+}$and $B_{L}$ employed in Sec. III are summarized. A full derivation of these expressions has been given elsewhere. ${ }^{19}$

The coefficients $A_{L}^{+}$are implicitly defined by

$$
\int d t e^{i \omega t} h_{L}^{(+)}[k(b, 0, \mathrm{v} t)]=i^{l} \frac{A_{L}^{+}}{\omega} K_{m}\left(\frac{\omega b}{\mathrm{v} \gamma}\right),
$$

where $\gamma=1 / \sqrt{1-(\mathrm{V} / c)^{2}}, \quad k=\omega / c$, and they can be calculated for $m \geqslant 0$ using the series ${ }^{19}$ 


$$
A_{L}^{+}=\left(\frac{c}{\mathrm{v}}\right)^{l+1} \sum_{j=m}^{l} \frac{C_{j}^{L,+}}{\gamma^{j}}
$$

(with $j+m$ even) where

$$
\begin{aligned}
C_{j}^{L,+}= & \frac{i^{l-j}(2 l+1) ! !}{2^{j}(l-j) ![(j-m) / 2] ![(j+m) / 2] !} \\
& \times \sqrt{[(2 l+1) /(4 \pi)](l-m) ! /(l+m) !} I_{j, l-j}^{L} .
\end{aligned}
$$

The numbers $I_{j, l-j}^{L}$ are obtained from the recurrence relation

$$
(l-m) I_{i_{1} i_{2}}^{L}=(2 l-1) I_{i_{1}, i_{2}+1}^{l-1, m}-(l+m-1) I_{i_{1}, i_{2}}^{l-2, m},
$$

valid for $l>m$, starting from the values $I_{i_{1} i_{2}}^{m-1, m}=0$ and

$$
I_{i_{1} i_{2}}^{m m}=\frac{1+(-1)^{i_{2}}}{2}(-1)^{m}(2 m-1) ! ! B\left(\frac{i_{1}+m+2}{2}, \frac{i_{2}+1}{2}\right) .
$$

Here $B$ is the beta function. ${ }^{47}$ For $m<0$, one can use the relation $A_{l,-m}^{+}=(-1)^{m} A_{L}^{+}$. Table I contains explicit expressions of $A_{L}^{+}$for the lowest values of $l$.

The coefficients $B_{L}$ are defined as

$$
\begin{aligned}
B_{L}= & A_{l, m+1}^{+} \sqrt{(l+m+1)(l-m)} \\
& -A_{l, m-1}^{+} \sqrt{(l-m+1)(l+m)} .
\end{aligned}
$$

Explicit expressions of $B_{L}$ for the lowest values of $l$ are given in Table II.
*Permanent address: Departamento de CCIA (Facultad de Informática), Donostia International Physics Center (DIPC), and Centro Mixto CSIC-UPV/EHU, San Sebastián, Spain.

${ }^{1}$ P. D. Nellist and S. J. Pennycook, Phys. Rev. Lett. 81, 4156 (1998).

${ }^{2}$ L. D. Marks, Solid State Commun. 43, 727 (1982).

${ }^{3}$ J. M. Cowley, Phys. Rev. B 25, 1401 (1982).

${ }^{4}$ A. Howie and R. H. Milne, Ultramicroscopy 18, 427 (1985).

${ }^{5}$ A. Howie and C. Walsh, Microsc. Microanal. Microstruct. 2, 171 (1991).

${ }^{6}$ D. W. McComb and A. Howie, Nucl. Instrum. Methods Phys. Res. B 96, 569 (1995).

${ }^{7}$ R. D. Averitt, D. Sarkar, and N. J. Halas, Phys. Rev. Lett. 78, 4217 (1997).

${ }^{8}$ R. H. Ritchie, Phys. Rev. 106, 874 (1957).

${ }^{9}$ P. M. Echenique and J. B. Pendry, J. Phys. C 8, 2936 (1975).

${ }^{10}$ E. Kröger, Z. Phys. A 235, 403 (1970).

${ }^{11}$ R. García-Molina, A. Gras-Martí, A. Howie, and R. H. Ritchie, J. Phys. C 18, 5335 (1985).

${ }^{12}$ N. Zabala, A. Rivacoba, and P. M. Echenique, Surf. Sci. 209, 465 (1989).

${ }^{13}$ M. Schmeits, Phys. Rev. B 39, 7567 (1989).

${ }^{14}$ R. García-Molina, A. Gras-Martí, and R. H. Ritchie, Phys. Rev. B 31, 121 (1985).

${ }^{15}$ R. Fuchs and K. L. Kliewer, J. Opt. Soc. Am. 58, 319 (1968).

${ }^{16}$ T. L. Ferrell and P. M. Echenique, Phys. Rev. Lett. 55, 1526 (1985).

${ }^{17}$ T. L. Ferrell, R. J. Warmack, V. E. Anderson, and P. M. Echenique, Phys. Rev. B 35, 7365 (1987).

${ }^{18}$ P. M. Echenique, A. Howie, and D. J. Wheatley, Philos. Mag. B 56, 335 (1987).

${ }^{19}$ F. J. García de Abajo, Phys. Rev. B 59, 3095 (1999).

${ }^{20}$ N. Zabala, A. Rivacoba, and P. M. Echenique, Phys. Rev. B 56, 7623 (1997).

${ }^{21}$ B. L. Illman, V. E. Anderson, R. J. Warmack, and T. L. Ferrell, Phys. Rev. B 38, 3045 (1988).

${ }^{22}$ A. Rivacoba, N. Zabala, and P. M. Echenique, Phys. Rev. Lett. 69, 3362 (1992).

${ }^{23}$ A. Rivacoba, J. Aizpurua, and N. Zabala, Scanning Microsc. 9, 927 (1995).

${ }^{24}$ J. Aizpurua, A. Rivacoba, and S. P. Apell, Phys. Rev. B 54, 2901 (1996).
${ }^{25}$ R. G. Barrera and R. Fuchs, Phys. Rev. B 52, 3256 (1995).

${ }^{26}$ C. I. Mendoza, R. G. Barrera, and R. Fuchs, Phys. Rev. B 57, 11193 (1998).

${ }^{27}$ L. Fu, P. B. Macedo, and L. Resca, Phys. Rev. B 47, 13818 (1993).

${ }^{28}$ L. Fu and L. Resca, Phys. Rev. B 47, 16194 (1993).

${ }^{29}$ F. Ouyang and M. Isaacson, Philos. Mag. B 60, 481 (1989).

${ }^{30} \mathrm{~F}$. Ouyang and M. Isaacson, Ultramicroscopy 31, 345 (1989).

${ }^{31}$ F. J. García de Abajo and J. Aizpurua, Phys. Rev. B 56, 15873 (1997).

${ }^{32}$ J. Aizpurua, B. Rafferty, F. J. García de Abajo, and A. Howie, in Electron Microscopy and Analysis Group Conference EMAG97, edited by C. J. Kiely, IOP Conf. Proc. No. 153 (Institute of Physics and Physical Society, London, 1997), Sec. 8, p. 277.

${ }^{33}$ J. Aizpurua, A. Howie, and F. J. García de Abajo, Phys. Rev. B (to be published 15 October 1999).

${ }^{34}$ J. B. Pendry and A. MacKinnon, Phys. Rev. Lett. 69, 2772 (1992).

${ }^{35}$ J. B. Pendry and L. Martín-Moreno, Phys. Rev. B 50, 5062 (1994).

${ }^{36}$ F. J. García-Vidal, J. M. Pitarke, and J. B. Pendry, Phys. Rev. Lett. 78, 4289 (1997).

${ }^{37}$ F. J. García de Abajo and A. Howie, Phys. Rev. Lett. 80, 5180 (1998).

${ }^{38}$ F. J. García de Abajo and A. Howie (unpublished).

${ }^{39}$ F. E. Low, Classical Field Theory: Electromagnetism and Gravitation (John Wiley \& Sons, Inc., New York, 1997).

${ }^{40}$ F. J. García de Abajo, Phys. Rev. B 60, 6086 (1999).

${ }^{41}$ A. Messiah, Quantum Mechanics (North-Holland, New York, 1966).

${ }^{42}$ F. J. García de Abajo, Phys. Rev. Lett. 82, 2776 (1999).

${ }^{43} R_{\alpha \beta}$ is defined as the rotation that brings the bond vector $\mathbf{d}_{\alpha \beta}$ $=\mathbf{r}_{\alpha}-\mathbf{r}_{\beta}$ into the positive $z$ direction, and it acts on the coefficients of the multipole expansions with Euler angles $(0, \theta, \pi$ $-\varphi)$, where $(\theta, \varphi)$ are the polar angles of $\mathbf{d}_{\alpha \beta}$.

${ }^{44}$ J. B. Pendry, Low Energy Electron Diffraction (Academic Press, London, 1974).

${ }^{45}$ E. D. Palik, Handbook of Optical Constants of Solids (Academic Press, New York, 1985).

${ }^{46}$ H. Li, B. Cheng, and D. Zhang, Phys. Rev. B 56, 10734 (1997).

${ }^{47}$ M. Abramowitz and I. A. Stegun, Handbook of Mathematical Functions (Dover, New York, 1972). 